\title{
Multiscale Interactions between Monsoon Intraseasonal Oscillations and Low Pressure Systems That Produce Heavy Rainfall Events of Different Spatial Extents
}

\author{
Akshaya C. Nikumbh, ${ }^{\mathrm{a}, \mathrm{b}}$ Arindam Chakraborty, ${ }^{\mathrm{a}, \mathrm{b}}$ G. S. Bhat, ${ }^{\mathrm{a}, \mathrm{b}}$ AND Dargan M. W. Frierson ${ }^{\mathrm{c}}$ \\ ${ }^{a}$ Centre for Atmospheric and Oceanic Sciences, Indian Institute of Science, Bangalore, India \\ ${ }^{\mathrm{b}}$ Divecha Centre for Climate Change, Indian Institute of Science, Bangalore, India \\ ${ }^{\mathrm{c}}$ Department of Atmospheric Sciences, University of Washington, Seattle, Washington
}

(Manuscript received 23 March 2021, in final form 3 August 2021)

\begin{abstract}
The subseasonal and synoptic-scale variability of the Indian summer monsoon rainfall is controlled primarily by monsoon intraseasonal oscillation (MISO) and low pressure systems (LPSs), respectively. The positive and negative phases of MISO lead to alternate epochs of above-normal (active) and below-normal (break) spells of rainfall. LPSs are embedded within the different phases of MISO and are known to produce heavy precipitation events over central India. Whether the interaction with the MISO phases modulates the precipitation response of LPSs, and thereby the characteristics of extreme rainfall events (EREs), remains unaddressed in the available literature. In this study, we analyze the LPSs that produce EREs of various spatial extents (small, medium, and large) over central India from 1979 to 2012 . We also compare them with the LPSs that pass through central India and do not produce any ERE (LPS-noex). We find that thermodynamic characteristics of LPSs that trigger different spatial extents of EREs are similar. However, they show differences in their dynamic characteristics. The ERE-producing LPSs are slower, moister, and more intense than LPSnoex. The LPSs that lead to medium and large EREs tend to occur during the positive phase of MISO when an active monsoon trough is present over central India. On the other hand, LPS-noex and the LPSs that trigger small EREs occur mainly during the neutral or negative phases of the MISO. The large-scale dynamic forcing, intensification of LPSs, and diabatic generation of low-level potential vorticity due to the presence of active monsoon trough help in the organization of convection and lead to medium and large EREs. On the other hand, the LPSs that form during the negative or neutral phases of MISO do not intensify much during their lifetime and trigger scattered convection, leading to EREs of small size.
\end{abstract}

KEYWORDS: Intraseasonal variability; Extreme events; Monsoons

\section{Introduction}

Although there have been significant advancements in the understanding of rainfall extremes over the past couple of decades, predicting their location and intensity with high accuracy and sufficient lead time has remained a challenging task. The challenges come mainly from identifying favorable initial conditions, feedbacks, external drivers, and nonlinear processes that lead to the development of extreme events (Sillmann et al. 2017). The interaction of several spatiotemporal scales during the Indian summer monsoon (Ding 2007; Singh et al. 2019) poses unique challenges to decipher these processes.

During the Indian summer monsoon, the subseasonal variability comes mainly from the monsoon intraseasonal oscillation (MISO) (Goswami and Mohan 2001; Webster et al. 1998; Wang 2006). The MISO consists of two dominant modes, 10 20-day (Krishnamurti and Bhalme 1976) and 30-60-day oscillations (Yasunari 1979; Sikka and Gadgil 1980), that account for $25 \%$ and $66 \%$ of total intraseasonal variability, respectively

Supplemental information related to this paper is available at the Journals Online website: https://doi.org/10.1175/JCLI-D-210231.s1.

Corresponding author: Akshaya C. Nikumbh, nikumbh@iisc. ac.in
(Annamalai and Slingo 2001). The 30-60-day mode, which originates over the equatorial Indian Ocean, travels northward to the Indian landmass, whereas the 10-20-day mode originates over the South China Sea and propagates northwestward (Webster et al. 1998; Wang 2006). The different phases of these two modes and their interactions lead to alternate periods of above (active) and below (break) normal rainfall over India. Conditions conducive for extreme rainfall events (EREs) are formed when both modes of the MISO are in phase and exhibit simultaneous positive anomalies (Karmakar et al. 2015). Francis and Gadgil (2006) showed that EREs over the Western Ghats are associated with the northward propagating tropical convergence zone. Likewise, the maximum number of EREs over central India occur during an active phase of lowfrequency MISO (30-60-day mode) (Karmakar et al. 2015). With the changing global climate, these intraseasonal oscillations of the monsoon are expected to become more extreme and increase the likelihood of EREs over India (Turner and Slingo 2009). The signs of these changes are already becoming evident over the past couple of decades. The active spells are becoming intense and the dry spells more frequent (Singh et al. 2014). The influence of the MISO on EREs over India has been considered before (Turner and Slingo 2009; Francis and Gadgil 2006; Singh et al. 2014; Karmakar et al. 2015), but its exact impact on the characteristics of EREs has not been elucidated in detail.

Embedded within the intraseasonal oscillations are synoptic disturbances called monsoon low pressure systems (LPSs). 
LPSs form mainly over the Bay of Bengal and travel northwestward, bringing the episodes of heavy rainfall over central India (Godbole 1977; Sikka 1977; Patwardhan et al. 2020). Ajayamohan et al. (2010) defined a normalized index called the synoptic activity index using the intensity, frequency, and duration of LPSs. They showed that the number of EREs over central India and the synoptic activity index show a strong correlation, and suggested that the increasing synoptic activity possibly explains the rising trend of EREs. Similarly, Karmakar et al. (2015) found that the relative contribution from the synoptic variability (3-9 days) to the variance of daily rainfall over central India has been increasing. Nikumbh et al. (2020) showed that two-thirds of LPSs that travel through central India give at least one ERE. They also showed that EREs with a large spatial extent (area $\geq 70000 \mathrm{~km}^{2}$ ) are initiated by the interaction between an LPS and a secondary cyclonic vortex (SCV). When an SCV is located to the west of an LPS, the conditions favorable for large-scale EREs are formed in between these two cyclonic vortices. Studies attribute either an increase in the number of LPS days (Pai et al. 2016) or their frequency of occurrence (Krishnamurthy 2012) to an increase in the frequency of EREs over central India. It has been shown that the different phases of MISO influence the formation and propagation of LPSs (Goswami et al. 2003). Goswami et al. (2003) observed the clustering of LPSs during an active phase of MISO. They showed that LPSs are 3.5 times more likely to form during the active phase of MISO than the break phase. Also, the tracks of LPSs during the active phase congregate mainly along the monsoon trough that is situated over central India. On the other hand, the LPSs that form during the break phase are confined to the Himalayan foothills. Does the interaction of the MISO and LPSs also play a role during EREs? The available literature does not address this question. The characteristics of LPSs, large-scale conditions, and pathways through which LPS and MISO might influence the spatial characteristics of EREs have not been explored yet.

Nikumbh et al. (2019) showed that EREs over central India exhibit different spatial scales and classified them as small, medium, and large EREs. They observe that the daily EREs of all sizes show a strong association with LPSs and occur mainly to the west of their center. They also noted that EREs of different sizes have different large-scale conditions and speculated that the underlying physical processes could be different for various sizes of EREs. Using a $k$-means clustering on hourly rainfall data, Moron et al. (2021) classified wet events over India into different storm types and grouped them into two broad categories. The first group with short space-time scales and intense rainfall is phase-locked with the diurnal cycle (i.e., triggered mostly in the midafternoon). The other group, with a longer duration, larger spatial extent, and less intense rainfall, does not show any peak triggering time, so is possibly associated with large mesoscale systems or tropical lows. These studies show that the governing processes of EREs may differ based on the spatial extent of EREs, which requires further investigation. The flooding susceptibility of a region is directly proportional to the spatial extent of EREs. The observed trends for EREs of different sizes are distinct. For example, large EREs are increasing significantly (Roxy et al. 2017;
Nikumbh et al. 2019). However, small-scale events do not show any trend (Nikumbh et al. 2019). Thus, understanding the underlying mechanism leading to EREs of different spatial scales will help to understand their observed trends, future projections, and socioeconomic impacts a step further.

In this study, our main objective is to understand the features and meteorological conditions that determine the response of LPSs while producing EREs of different spatial extents (small, medium, and large) over central India. We speculate that the different phases of MISO could modulate the precipitation response of LPSs. Thus, we explore the possible influence of the MISO phases on the spatial extent of EREs produced by LPSs. The manuscript is structured as follows: section 2 discusses data and methods. Section $3 \mathrm{a}$ and $3 \mathrm{~b}$ examine the characteristics and background conditions of ERE producing LPSs, respectively. Sections $3 \mathrm{c}-3 \mathrm{e}$ explain physical processes through which the characteristics and background conditions help LPSs produce EREs of different spatial extents. Section 4 provides a summary and discussion.

\section{Method}

EREs of the summer monsoon months (June to September) for the period of 1979-2012 are identified using the daily gridded rainfall dataset developed by the India Meteorological Department (Rajeevan et al. 2006). This dataset has a spatial resolution of $1^{\circ} \times 1^{\circ}$. Central India $\left(15^{\circ}-25^{\circ} \mathrm{N}, 75^{\circ}-85^{\circ} \mathrm{E}\right.$; inset box in Fig. 1a) is our study area.

Following Nikumbh et al. (2019), an ERE at each grid is identified using the 99.5 percentile threshold; then, using the connected component labeling analysis (Falcão et al. 2004), the neighboring grids with extreme rainfall are combined. This gives EREs of different sizes, where the size is measured in $1^{\circ} \times 1^{\circ}$ grid units. The EREs are then classified according to their spatial extents as small ( size $=1$; area $\leq 10^{4} \mathrm{~km}^{2}$ ), medium (size 2-5; $10^{4} \mathrm{~km}^{2} \leq$ area $\leq 7 \times 10^{4} \mathrm{~km}^{2}$ ), and large (size $\geq 6$; area $\geq 7 \times 10^{4} \mathrm{~km}^{2}$ ) EREs. Our classification of EREs into three types is based on the results reported in Nikumbh et al. (2019) and Nikumbh et al. (2020). It mainly takes the frequency of occurrence and synoptic signatures into account.

To avoid a possible repeat count of the same event, EREs are checked iteratively for two consecutive days. If any two EREs occur on consecutive days, the larger size ERE is considered and the smaller size ERE is discarded. If an ERE of the same category occurs on the next day, then only an ERE of the first day is considered. Over central India, the probability of getting small EREs is the highest (probability $=71.4 \%$; Fig. 1a) and this probability decreases as the size of ERE increases. The average probability of occurrences of medium and large EREs are $26.2 \%$ and $2.4 \%$, respectively. Medium and large events are not only bigger in the spatial extent but also slightly more intense (Fig. 1b). The average rainfall at the center of small, medium, and large EREs is 122, 135, and $145 \mathrm{~mm} \mathrm{day}^{-1}$, respectively.

The dataset by Hurley and Boos (Hurley and Boos 2015), which is available from 1979 to 2012, is used to identify LPSs. The presence of an LPS is checked for a given day of an ERE 
(a)

EREs of central India

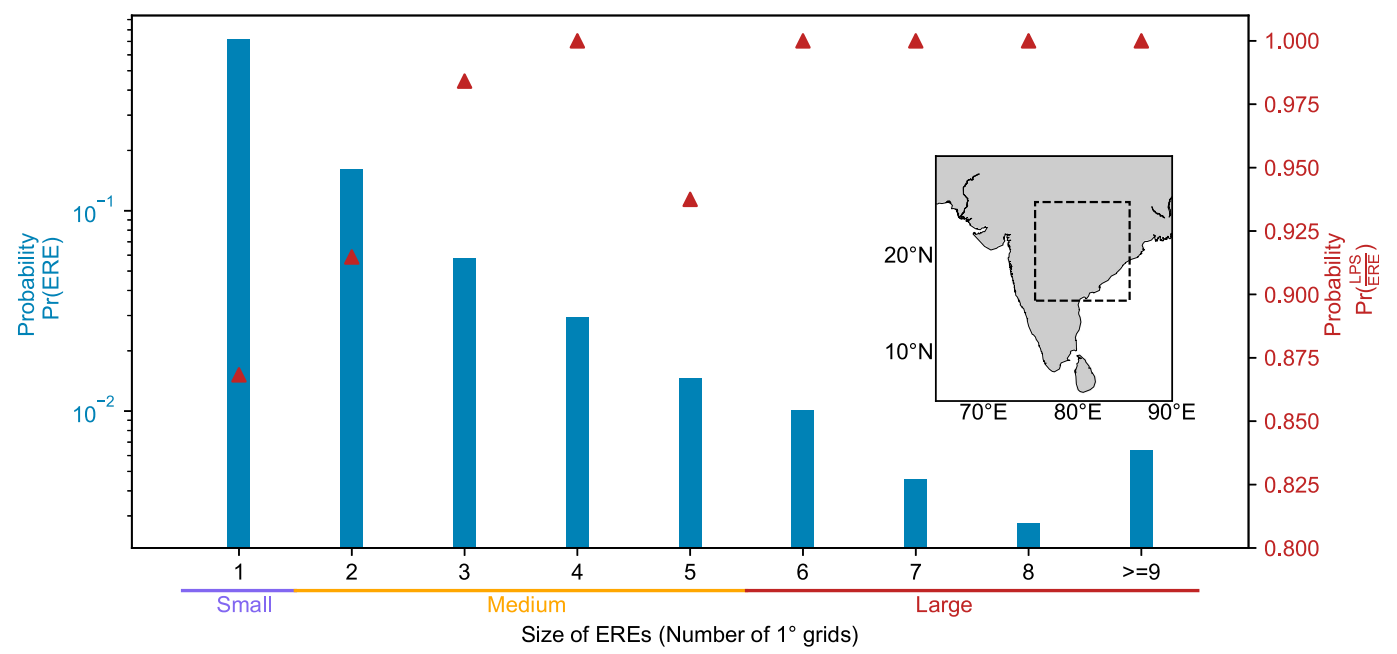

(b)
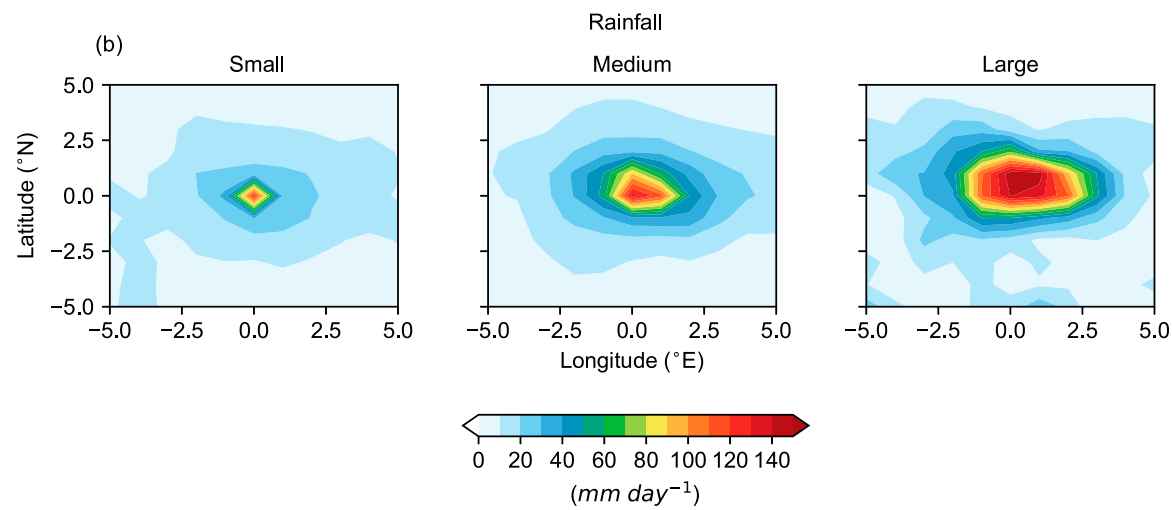

FIG. 1. (a) The probability of occurrence of extreme rainfall events (EREs) [Pr (ERE); bars] and the probability of a presence of an LPS for EREs of different sizes [Pr(LPS/ERE); brown markers], where the size is measured in $1^{\circ} \times 1^{\circ}$ grid units. Based on the size, EREs are classified as small (size $=1$ ), medium (size $=2-5$ ), and large (size $\geq 6$ ). Note the logarithmic scale for the probability of occurrence. The probabilities for size $\geq 9$ are clubbed in the last bar. (b) Rainfall intensity $\left(\mathrm{mm} \mathrm{day}^{-1}\right)$ distribution with respect to the geometric center of EREs. The inset box in Fig. 1a shows the study region (central India: $15^{\circ}-25^{\circ} \mathrm{N}, 75^{\circ}-85^{\circ} \mathrm{E}$ ).

over the domain of $75^{\circ}-90^{\circ} \mathrm{E}, 15^{\circ}-30^{\circ} \mathrm{N}$. As shown in Fig. 1a, EREs of central India show a strong association with LPSs. EREs of all sizes over central India have an LPS present for more than $80 \%$ of the time. During the study period, we observe that all Large EREs were associated with LPSs. If there are multiple LPSs on the same day, only the nearest LPS to an ERE is considered for the analysis. To address our objective, we consider only those EREs that had an LPS present. We also examined the LPSs that did not produce any ERE over central India to bring out the contrast. The LPSs that lead to small, medium, and large EREs are referred to as LPS-sm [number of cases $(n)=118]$, LPS-med $(n=133)$, and LPS-lg $(n=20)$, respectively (abbreviations listed in Table 1). The LPSs that pass through the study region and did not lead any ERE are named as LPS-noex $(n=166)$.

The meteorological conditions are studied using the ERAInterim reanalysis (Dee et al. 2011). The lag composites are calculated with respect to the day of an ERE (day 0) for ERE producing LPSs. For LPS-noex, day 0 indicates the genesis day of an LPS. The daily anomalies of meteorological fields are obtained by subtracting the daily climatology. The significance of anomalies is checked using the two-sided $t$ test. The cumulative distribution functions (CDFs) and boxplots are compared using the Kolmogorov-Smirnov test (K-S test). The 95\% confidence intervals for the line plots are calculated by generating $10^{4}$ bootstrapping samples.

\section{Results}

\section{a. Characteristics}

The characteristics of LPSs such as relative vorticity, speed of propagation, and total column water vapor are evaluated along their entire path at a 6-hourly time step (Fig. 2). The relative vorticity ( $\zeta$ at $850 \mathrm{hPa}$ ) is lowest for LPS-noex (average $=4 \times 10^{-5} \mathrm{~s}^{-1}$ ) and gradually increases 
TABLE 1. List of abbreviations.

\begin{tabular}{ll}
\hline \hline Abbreviations & \multicolumn{1}{c}{ Expansion } \\
\hline MISO & Monsoon intraseasonal oscillation \\
LPS & Low pressure system \\
ERE & Extreme rainfall event \\
LPS-noex & Low pressure system that does not produce \\
& any extreme rainfall event \\
LPS-sm & Small size extreme rainfall event producing \\
& low pressure system \\
LPS-med & Medium size extreme rainfall event \\
& producing low pressure system \\
LPS-lg & Large size extreme rainfall event producing \\
& low pressure system \\
SCV & Secondary cyclonic vortex \\
LLJ & Low-level jet \\
\hline
\end{tabular}

from LPS-sm (average $\left.=5 \times 10^{-5} \mathrm{~s}^{-1}\right)$ to LPS-med (average $=$ $6 \times 10^{-5} \mathrm{~s}^{-1}$ ), and finally is the highest for LPS-lg (average $=$ $\left.8 \times 10^{-5} \mathrm{~s}^{-1}\right)$. The LPSs that produce EREs have a significantly slower speed of propagation than LPS-noex ( $p$ value $<$ $0.01)$. Although LPS-sm tend to be faster, the speed of propagation of ERE-producing LPSs is not much different from each other $(p$ value $>0.05)$. The average speed of ERE producing LPSs is $4.6 \mathrm{~m} \mathrm{~s}^{-1}$, whereas LPS-noex have an average speed of $5.3 \mathrm{~m} \mathrm{~s}^{-1}$. LPS-noex are significantly drier than ERE producing LPSs and they get progressively wetter from LPSsm to LPS-lg ( $p$ value for each pair of LPS $<0.01$ ). The average total column water vapor of LPS-noex, LPS-sm, LPS-med, and LPS- $\lg$ is $51,61,63$, and $65 \mathrm{~kg} \mathrm{~m}^{-1}$, respectively.

Further, we look at the distribution of pressure velocity ( $\omega$ at $500 \mathrm{hPa})$, relative humidity $(\mathrm{RH})$, and equivalent potential temperature $\left(\theta_{e}\right)$ (Fig. 3). The absolute value of $\omega$ increases progressively from small to medium and is maximum for large EREs (Fig. 3a). The location of an ERE is marked by the region of maximum $\omega$, and its spread determines the spatial extent of ERE. In the coordinate system of an LPS, EREs are located to the western side of the center of an LPS (Nikumbh et al. 2019). Thus, the upward motion in the midtroposphere on the eastern side of EREs indicates the updraft associated with LPSs. For large EREs, the ascending zone is present even to the west of ERE due to the presence of an SCV (Nikumbh et al. 2020).

At low levels, the atmosphere is mostly saturated for all types of EREs (Fig. 3b). Below $700 \mathrm{hPa}$ to the west of EREs, the low-level jet (LLJ) (see supplemental Fig. 1a in the online supplemental material) provides the moisture that saturates the low-level atmosphere (supplemental Fig. 2). At upper levels, the air that comes from the dry northwest landmass of India is less humid. On the other hand, the air on the east side, which comes with LPSs from the Bay, is more humid even at upper levels. The interesting difference among different EREs is the presence of a deep saturated air layer that extends up to the midtroposphere near the center of medium and large EREs. The deep saturated layer helps to sustain the convection by providing higher plume buoyancies (Bretherton et al. 2004; Holloway and Neelin 2009). This deep humid layer is absent for small EREs. The spread of the saturated layer at low levels does not seem to be playing a role in determining the spatial extent of EREs. Similar to RH, the distribution of $\theta_{e}$ at $950 \mathrm{hPa}$ does not determine the extent of EREs (supplemental Fig. 3). The median value of $\theta_{e}$ and its distribution for all EREs is almost similar (Fig. 3c; $p$ value using the $\mathrm{K}-\mathrm{S}$ test $>0.05$ ). On the other hand, the distribution of $\omega$ at $500 \mathrm{hPa}$ is significantly different for all (Fig. 3d, $p$ value using the $\mathrm{K}-\mathrm{S}$ test $<0.05$ ) and its spread matches with the spatial extent of EREs (Fig. 3a).

The changes in precipitation are often investigated in terms of dynamic and thermodynamic characteristics (Trenberth et al. 2003; Allan and Soden 2008; Emori and Brown 2005). The circulation-related characteristics are known as dynamic characteristics (e.g., $\omega, u, v)$ and those related to temperature or moisture are called thermodynamic characteristics (e.g., RH, static stability). From the primary (a)

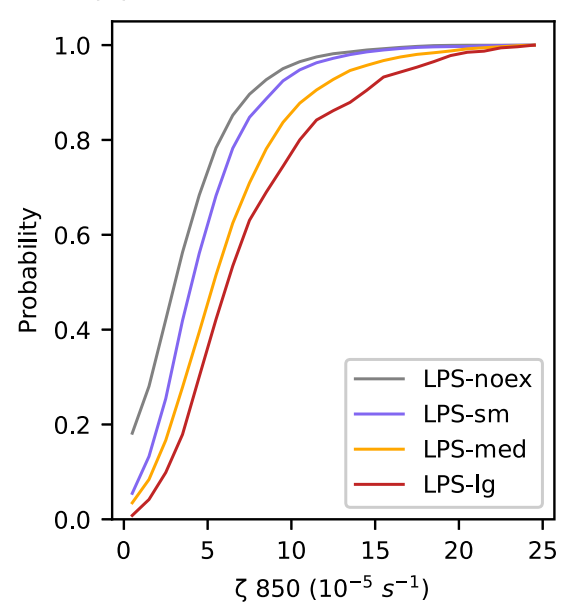

(b)

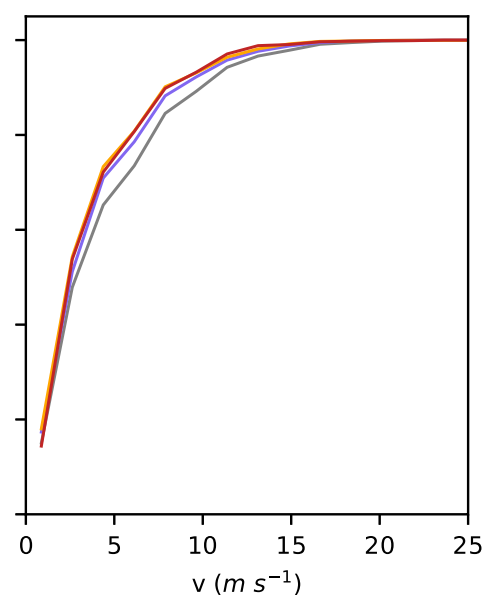

(c)

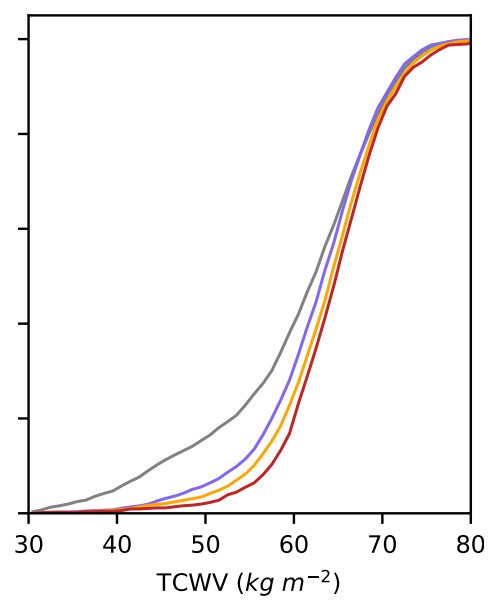

FIG. 2. Cumulative distribution function (CDF) of (a) relative vorticity at $850 \mathrm{hPa}\left(\zeta_{850}\right)$, (b) speed of propagation, and (c) total column water vapor (TCWV) for the LPSs that lead to EREs of different sizes-small (LPS-sm), medium (LPS-med), and large (LPS-lg)—and the LPSs that did not lead any ERE over central India (LPS-noex). The 6-hourly values along the tracks of LPSs are used to calculate the CDFs. 


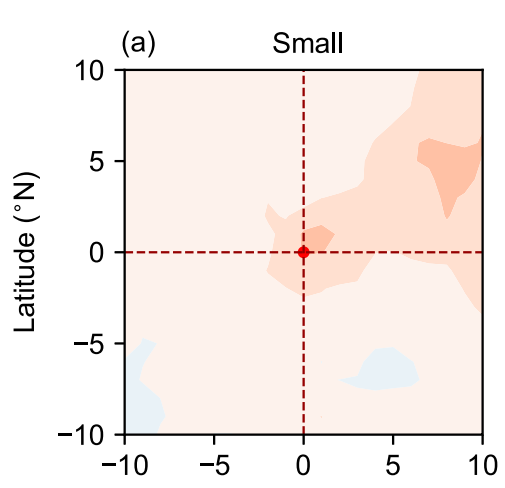

(b)

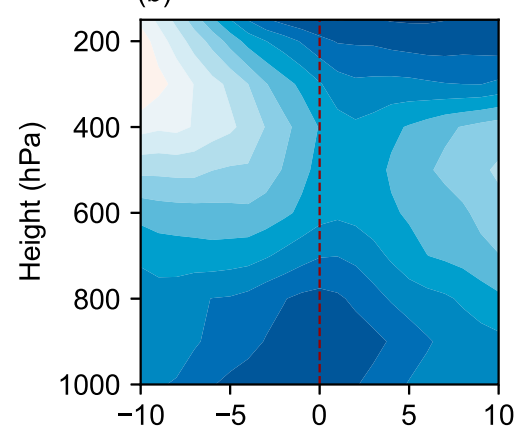

$\omega 500$

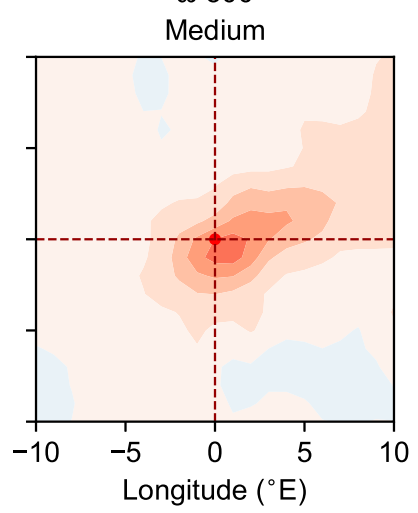

RH

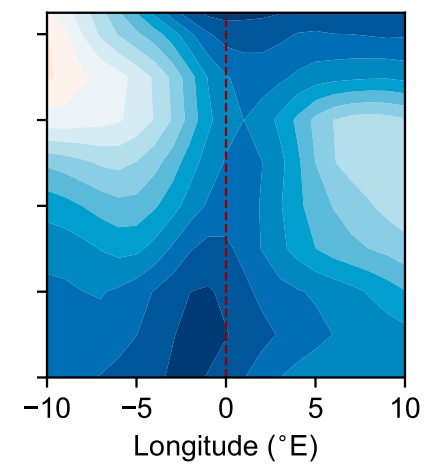

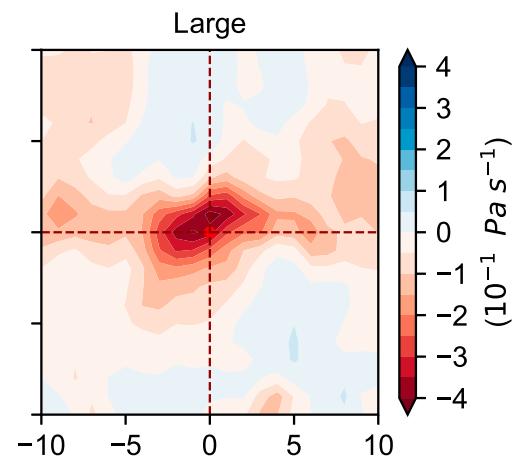

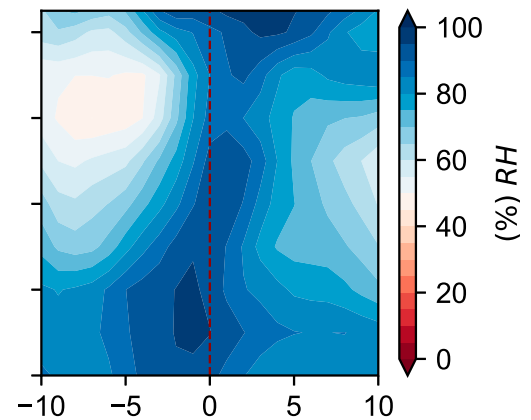

(c)

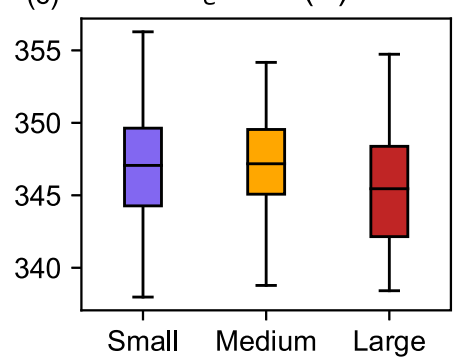

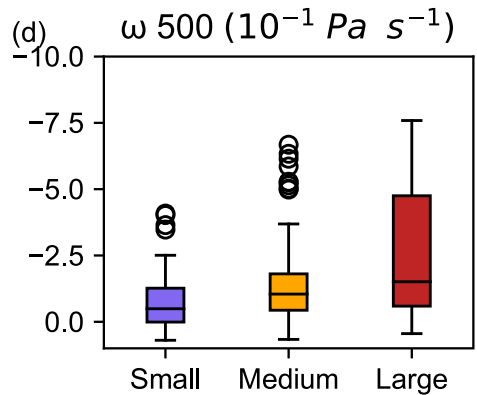

FIG. 3. Distribution of the daily composite (a) pressure velocity at $500 \mathrm{hPa}(\omega 500)$ with respect to the geometric center of EREs and (b) relative humidity $(\mathrm{RH})$ in the longitude-height plane along the latitude of EREs (the red dashed horizontal line in Fig. 3a). The red dashed horizontal and vertical lines pass through the center of ERE. Also shown are box plots of the average (c) equivalent potential temperature at $950 \mathrm{hPa}\left(\theta_{e} 950\right)$ and $(\mathrm{d}) \omega$ at $500 \mathrm{hPa}(\omega 500)$. The averages of $\theta_{e} 950$ and $\omega 500$ are calculated over $2^{\circ} \times 2^{\circ}$ latitude-longitude box around the geometric center of EREs. The horizontal black lines in the box plot indicate the median values. The box shows the interquartile range. The whiskers and black circles denote the extreme values (nonoutliers) and outliers, respectively.

analysis, we see that the dynamic characteristics play a key role in determining the spatial extent of EREs, whereas the spread of thermodynamic quantities does not seem to be determining the location and spatial extent of EREs.

\section{b. Background conditions}

The lagged composites of geopotential height bring out the distinct background conditions for LPSs that lead to EREs of different spatial extents (Figs. 4a-c). Zonally elongated anomalies are observed from two days before the occurrence of large and medium EREs, indicating the active monsoon conditions. They are not seen prior to small EREs and during the genesis of LPS-noex (supplemental Fig. 4a). For large EREs, there is also a signature of an SCV over the west coast. During the break, rainfall over central India is subdued but it is enhanced over southeastern India (Ramamurthy 1969; Pai et al. 2016). It exhibits the anomalous low over the southeastern peninsula similar to the observed anomalies for LPS-sm (Fig. 4a) and LPS-noex (supplemental Fig. 4a). 
(a) Small Day(-2)

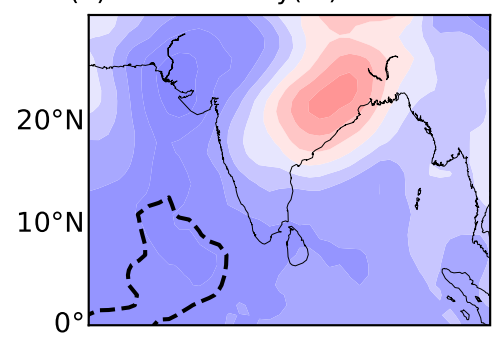

$\operatorname{Day}(-1)$

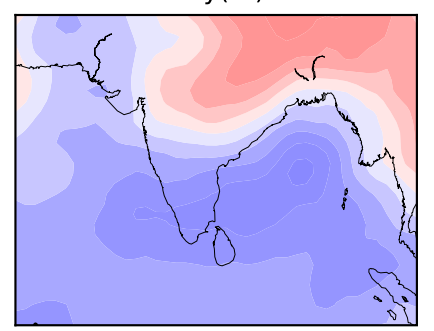

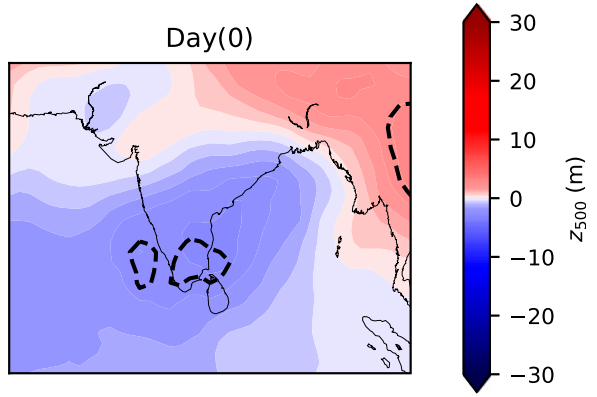

(b) Medium

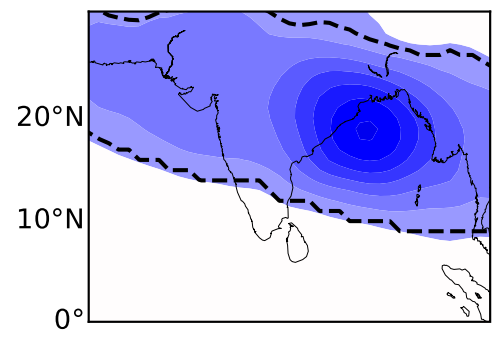

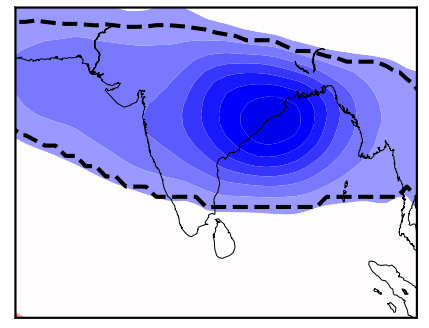

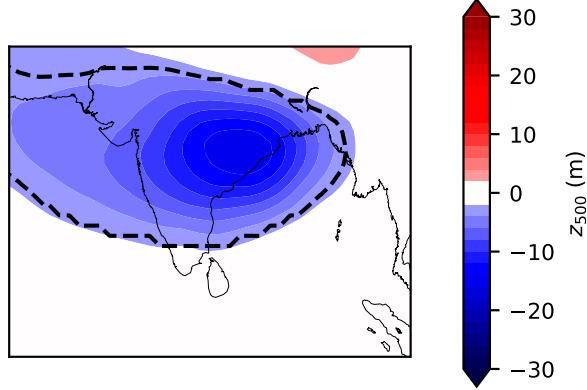

(c) Large

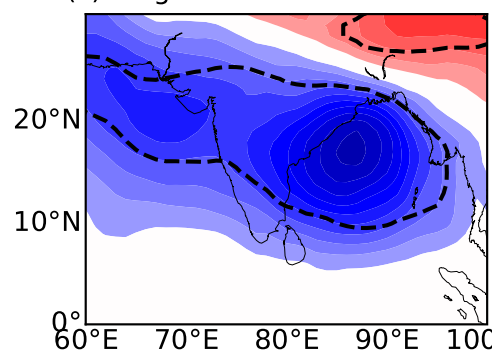

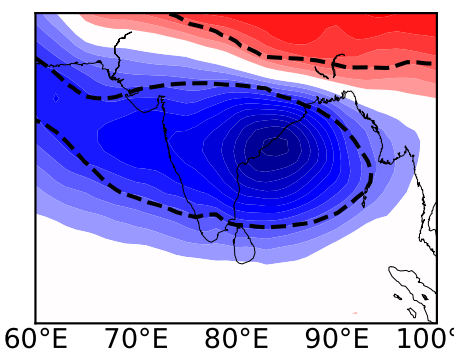

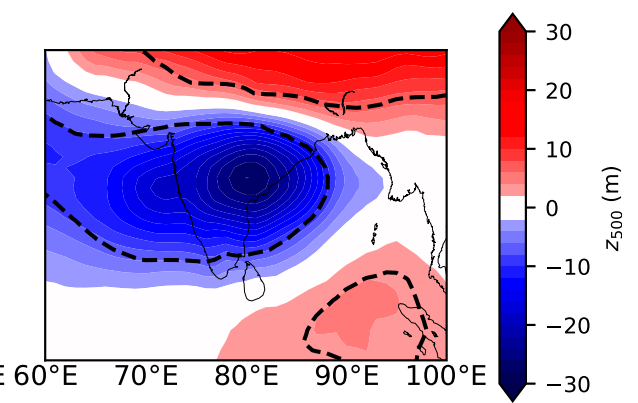

FIG. 4. Lagged composites of anomalous geopotential height (m) at $500 \mathrm{hPa}$ for (a) small, (b) medium, and (c) large EREs from day -2 to day 0 (shown from left to right). Day 0 indicates the day of an ERE. The dashed contour represents statistically significant anomalies of geopotential height $(t$ test: $p$ value $<0.05)$.

The presence of active monsoon conditions can be confirmed further by the rainfall over the core monsoon zone (Rajeevan et al. 2010). The rainfall anomalies are positive over the core monsoon zone before the occurrence of large and medium EREs (Fig. 5), indicating an active monsoon spell. The active spell during large EREs is more intense than that of medium EREs. For small EREs and LPS-noex, rainfall over central India is below or close to normal. On the day of ERE, the rainfall anomalies peak over central India and then slowly start falling. As day 0 for LPS-noex is the day of genesis, the peak in the rainfall anomalies over central India comes slightly later (day +3$)$, when the LPS makes landfall and travels over central India (supplemental Fig. 4b).

To confirm an association of EREs with the phase of the MISO, we define the MISO index following Goswami et al. (2003). The relative vorticity at $850 \mathrm{hPa}$ is averaged over the core monsoon zone $\left(75^{\circ}-90^{\circ} \mathrm{E}, 15^{\circ}-25^{\circ} \mathrm{N}\right)$ from May to October of each year from 1979 to 2012 . These anomalies are then detrended first and filtered for 10-90 days. To avoid the edge effects, we use the Tukey window. The filtered values are then normalized by the standard deviation of the relative vorticity for the entire time period to get the MISO index. The kernel density estimate of the MISO index is shown in Fig. 6. The LPS-lg and LPS-med show a peak at the positive MISO index while LPS-sm peaks at the negative MISO index. Respectively, $70 \%$ and $65 \%$ of LPS-lg and LPS-med occur during the positive phase of the MISO. On the other hand, $80 \%$ and $84 \%$ of LPS-sm and LPS-noex occur during the neutral to negative phase of the MISO (MISO index $\leq 0.5$ ). This consolidates our speculation of a positive MISO phase for LPS-lg and LPS-med, and a neutral or negative phase for LPS-sm and LPS-noex.

The preferential location where EREs of different sizes occur follows the location of the monsoon trough during the 


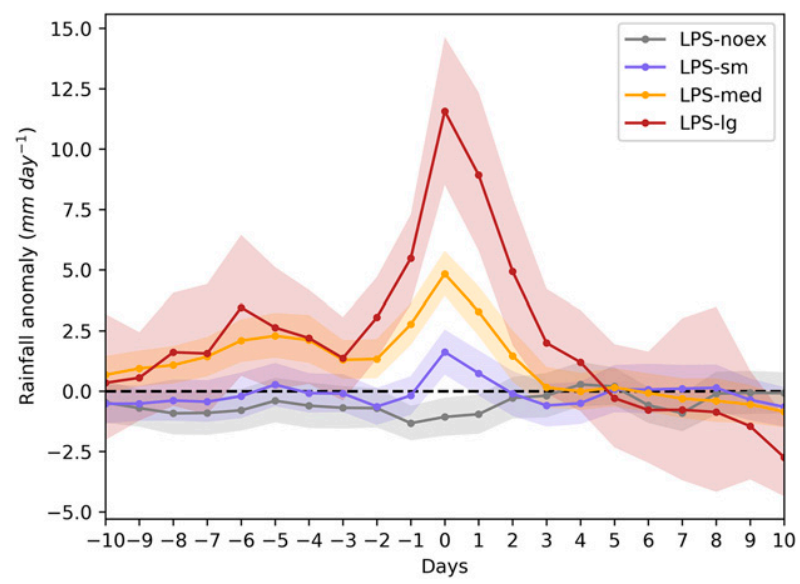

FIG. 5. Composite daily rainfall anomalies over the core monsoon zone $\left(15^{\circ}-25^{\circ} \mathrm{N}, 65^{\circ}-75^{\circ} \mathrm{E}\right)$ from lag days -10 to +10 for LPSsm, LPS-med, LPS-lg, and LPS-noex. The shading indicates the $95 \%$ confidence interval obtained by generating $10^{4}$ bootstrapping samples. The black dashed line indicates the zero line.

active and break phases of the MISO. The trough shifts northward near the foothills during the break phase, and it is situated over the core monsoon zone during an active phase (Rao 1976; Ramamurthy 1969). Small EREs tend to occur near the northern boundary of the study region (north of $22^{\circ} \mathrm{N}$ ), while medium to large EREs occur mainly in the southern part $\left(15^{\circ}-20^{\circ} \mathrm{N}\right)$ [Fig. $2 \mathrm{~b}$ and supplemental Fig. 5 of Nikumbh et al. (2019)]. This corroborates again the influence of the MISO on the different sizes of EREs.

The background conditions reveal that large and medium EREs occur mainly during the positive (active) phase of the MISO, while small EREs tend to occur during the neutral or negative phase of the MISO. By looking at the forcing responsible for the distribution of $\omega$ and the organization of convection, we can understand the processes that control the spatial extent of EREs. In the following sections, we analyze these processes by looking at dynamic forcing, vortex intensification, and diabatic generation of potential vorticity.

\section{c. Dynamic forcing}

By using the quasigeostrophic (QG) omega equation (Hoskins et al. 1978) we examine the dynamic forcing that leads to the observed differences in the distribution of vertical velocity. We use the modified definition of the Q-vector $(-2 \nabla \cdot \mathbf{Q})$ (Kiladis et al. 2006; Phadtare and Bhat 2019; Boos et al. 2015) for the tropics, where the geostrophic wind is replaced by the rotational wind. This modified form of $\nabla \cdot \mathbf{Q}$ is given by

$$
\begin{aligned}
\frac{p}{R} \nabla \cdot \mathbf{Q} \simeq & \frac{\partial T}{\partial x} \frac{\partial \zeta}{\partial y}-\frac{\partial T}{\partial y} \frac{\partial \zeta}{\partial x}-\frac{\partial^{2} T}{\partial x \partial y}\left(\frac{\partial^{2} \psi}{\partial^{2} x}-\frac{\partial^{2} \psi}{\partial^{2} y}\right) \\
& +\frac{\partial^{2} \psi}{\partial x \partial y}\left(\frac{\partial^{2} T}{\partial^{2} x}-\frac{\partial^{2} T}{\partial^{2} y}\right)
\end{aligned}
$$

where $p, R, T, \zeta$, and $\psi$ are pressure, the gas constant for dry air, temperature, the vertical component of relative vorticity, and the streamfunction of the horizontal wind, respectively; other

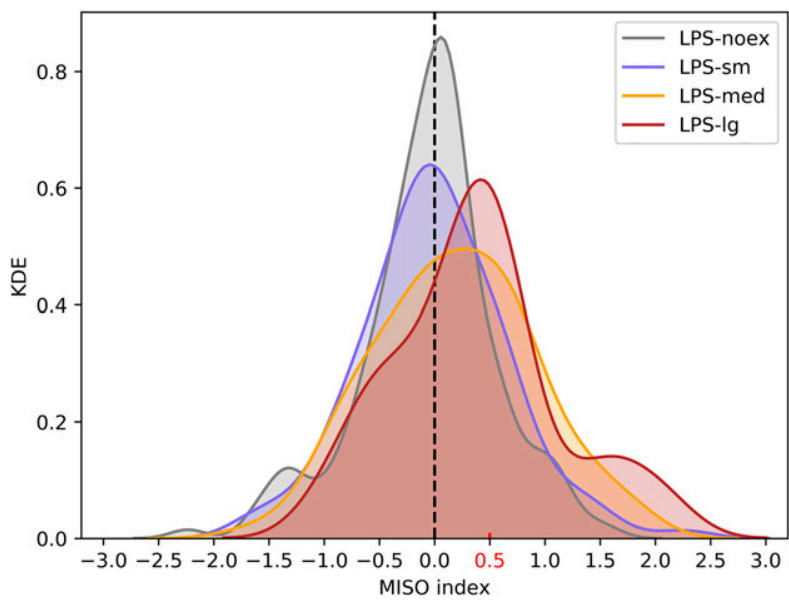

FIG. 6. Kernel density estimation (KDE) of the MISO index (defined in section 3b) on day 0 of LPS-sm, LPS-med, LPS-lg, and LPS-noex. The MISO index below 0.5 refers to the neutral to negative phase of the MISO.

terms have their usual meaning. Figure 7 shows the evolution of dynamic forcing $(-2 \nabla \cdot \mathbf{Q}$ at $500 \mathrm{hPa})$ over central India before and after the occurrence of EREs of different sizes. The large increase in the dynamic forcing over the entire central India is observed from two days before medium and large EREs. The enhanced dynamic forcing over central India for medium and large EREs is due to the presence of active monsoon conditions (Fig. 4). During an active monsoon, the enhanced relative vorticity over the core monsoon zone and the negative vorticity anomalies over the foothills exist, which are also observed for LPS-med and LPS-lg (supplemental Fig. 5). The presence of a zonally elongated monsoon trough enhances the vorticity and temperature (supplemental Fig. 5) that sets up large-scale dynamic forcing over central India for medium and large EREs. For LPS-sm and LPS-noex, the

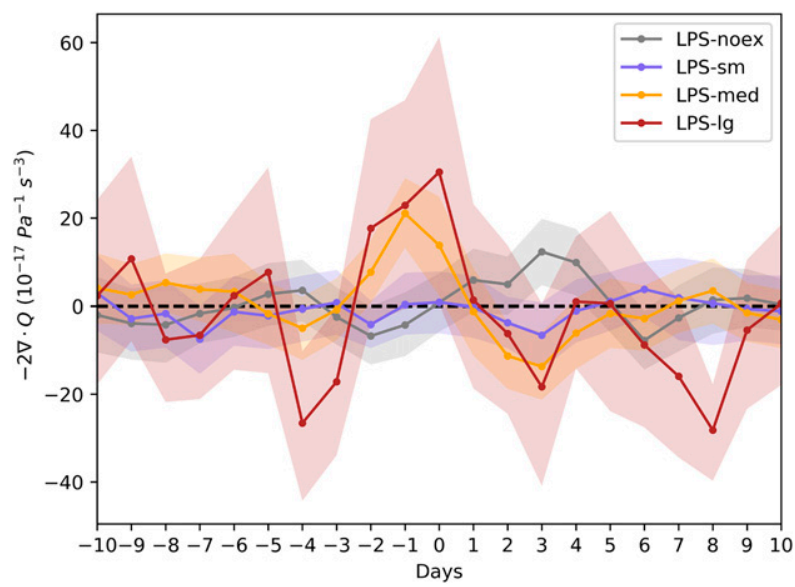

FIG. 7. Composite daily QG forcing $(-2 \nabla \cdot \mathbf{Q})$ over the study region from lag days -10 to +10 . The shading indicates the $95 \%$ confidence interval obtained by generating $10^{4}$ bootstrapping samples. 
(a)

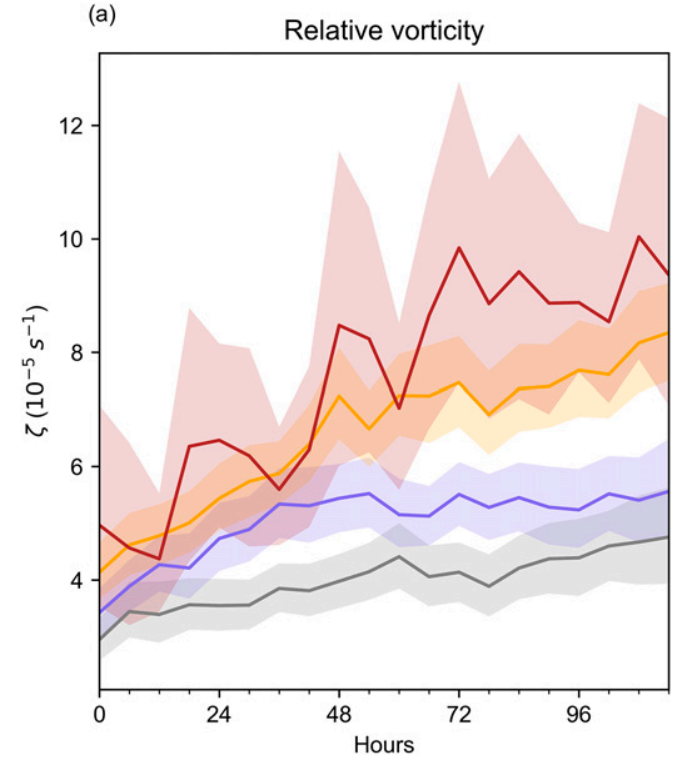

(b)

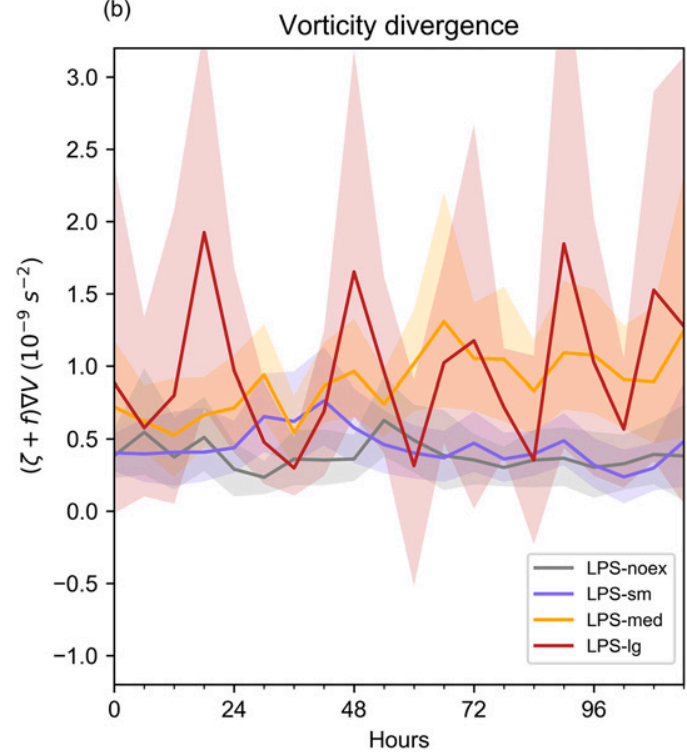

FIG. 8. Time evolution of (a) relative vorticity and (b) the vorticity divergence at $850 \mathrm{hPa}$. The 6-hourly terms are evaluated along the tracks of LPSs. The shading indicates the $95 \%$ confidence interval obtained by generating $10^{4}$ bootstrapping samples.

large-scale dynamic forcing is absent. For small EREs, the anticyclonic vorticity and cold anomalies are observed over central India, which creates weak dynamic subsidence over central India before the arrival of LPSs. When an LPS-sm enters central India large-scale dynamic forcing is absent, and it barely overcomes the background dynamic subsidence. The LPS-noex registers a slight increase in the dynamic forcing over central India, three days after its genesis, coincident with its landfall over central India.

\section{d. Vortex intensification}

LPS-lg and LPS-med are not only stronger compared to LPS-sm and LPS-noex during their genesis but also continue to intensify throughout their lifetime (Fig. 8a). The LPS-lg has the highest growth rate followed by LPS-med. The LPS-sm and LPS-noex have very small growth rates. The vortex stretching (vorticity divergence) is the major source of vorticity at low levels for LPSs (Daggupaty and Sikka 1977; Godbole 1978; Rajamani and Sikdar 1989). It is especially high for LPS-lg and LPS-med (Fig. 8b). During the positive phase of MISO, the strength of the LLJ is high and a strong wind exist over the southern boundary of the monsoon trough (supplemental Fig. 1a). The interaction of the LLJ with LPS-lg and LPS-med results in the formation of the strong convergence zone to the west of LPSs (supplemental Fig. 1b), which results in the vortex stretching and its intensification. This shows that the positive phase of MISO helps in the intensification of the LPS-lg and LPS-med. On the other hand, LPS-sm and LPS-noex do not intensify as much because the LLJ is weak during the negative and neutral phases of MISO.

\section{e. Diabatic generation of potential vorticity}

We look at the influence of an active monsoon trough in strengthening LPS-lg and LPS-med through the diabatic effects by examining potential vorticity (PV). An approximate diabatic generation of PV is given by (Martin 2006)

$$
\frac{d}{d t}(\mathrm{PV}) \approx-g(\zeta+f) \frac{\partial}{\partial p}\left(\frac{d \dot{Q}}{d t}\right),
$$

where PV, $g, \zeta, f$, and $d \dot{Q} / d t$ are potential vorticity, gravitational constant, relative vorticity, the Coriolis parameter, and diabatic heating, respectively. PV is generated where the vertical gradient of diabatic heating term is positive and destroyed where it is negative. We calculate the diabatic heating term $d \dot{Q} / d t$, which is a residual term of the thermodynamics equation (Holton and Hakim 2012; Ling and Zhang 2013) as follows:

$$
\left(\frac{d \dot{Q}}{d t}\right)=\frac{T}{\theta}\left(\frac{\partial \theta}{\partial t}+u \frac{\partial \theta}{\partial x}+v \frac{\partial \theta}{\partial y}+\omega \frac{\partial \theta}{\partial p}\right)
$$

The vertical profile of diabatic heating averaged over central India is shown from day -15 to day 0 in Fig. 9. About 15 days before EREs, the vertical profile of diabatic heating is almost same for all cases. As noted in the earlier studies, the midlevel warming is generated by convection (Ajayamohan et al. 2016; Hazra and Krishnamurthy 2015; Yanai et al. 1973). The lowlevel and upper-level cooling are associated with evaporative cooling and radiative cooling, respectively. The distinction among the profiles starts from day -9 when the rainfall anomalies (Fig. 5) over central India for large and medium EREs slowly start picking up. The midtropospheric diabatic heating continues to intensify for large and medium EREs in tandem with the rainfall anomalies over central India. The maximum anomaly in diabatic heating at midlevel is observed a day before 

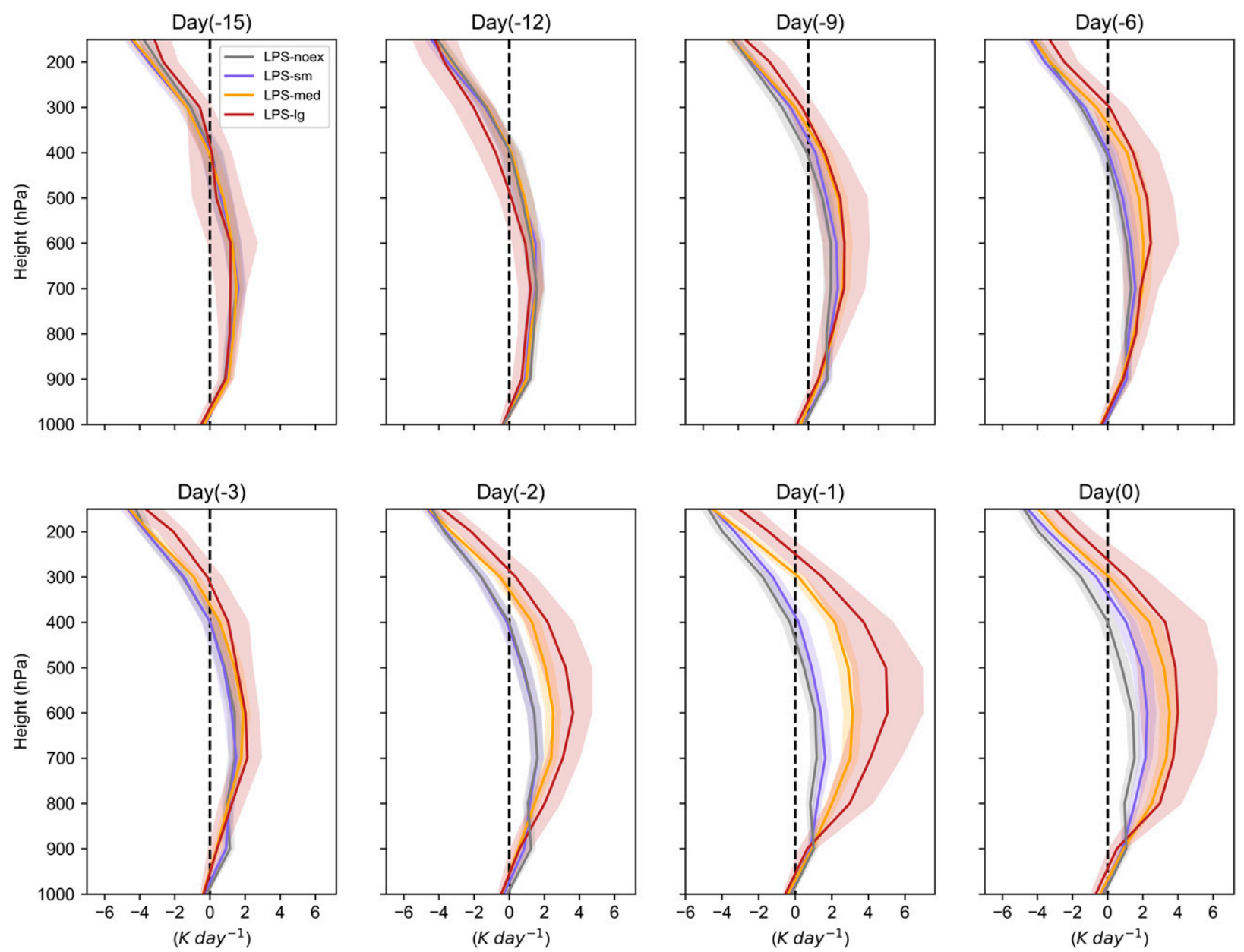

FIG. 9. Vertical profile of diabatic heating averaged over the study region from day -15 to day 0 . The shading represents $95 \%$ confidence interval obtained by generating $10^{4}$ bootstrapping samples.

large EREs. For medium EREs, the anomalies of diabatic heating are almost similar on the day of an ERE and a day before. For small EREs, they peak on the event day. The vertical gradient in the diabatic heating leads to a positive PV tendency in the lower troposphere and negative tendency in the upper troposphere (Fig. 10). A day before large and medium EREs, the diabatic PV generation deepens and reaches up to the midtroposphere. Hence, the cyclonic circulation at low to midlevels is strengthened over central India partly due to diabatic heating during the positive MISO phase for LPS-lg and LPSmed. This strengthened circulation can combine the scattered convection, and form a large-scale organized convection, leading to a large spatial extent of EREs.

\section{Summary and discussion}

In this study, we investigate the characteristics and background conditions of the monsoon lows (LPSs) that lead to extreme rainfall events (EREs) of different spatial extents: small ( size $=1$, LPS-sm), medium ( large (size $\geq 6$, LPS-lg) EREs over central India. We also compare them with the LPSs that do not lead to any ERE (LPS-noex).

The characteristics (section 3a) of these LPSs reveal that the ERE-producing LPSs are slower, moister, and more intense than the LPSs that do not give EREs (Fig. 2). The spatial extent and the location of EREs are coincident with the midtropospheric $\omega$ maximum. The spread of thermodynamic entities such as relative humidity or equivalent potential temperature does not seem to be controlling the spatial extent of EREs. This possibly indicates that the spatial extent of EREs is determined primarily by the dynamic characteristics (Fig. 3).

The analysis of background conditions (section 3b) shows that the LPSs that set off medium and large EREs occur mainly during the positive phase of the MISO (Figs. 4-6), which forms an active monsoon trough over central India. On the other hand, the LPSs that form during the negative and neutral phases of the MISO do not have an active monsoon trough over central India and produce small EREs. The LPSs that do not lead to any ERE also occur mainly during the neutral to negative phase of the MISO. As LPSs are embedded in the different phases of the MISO, we infer that it is the interaction 

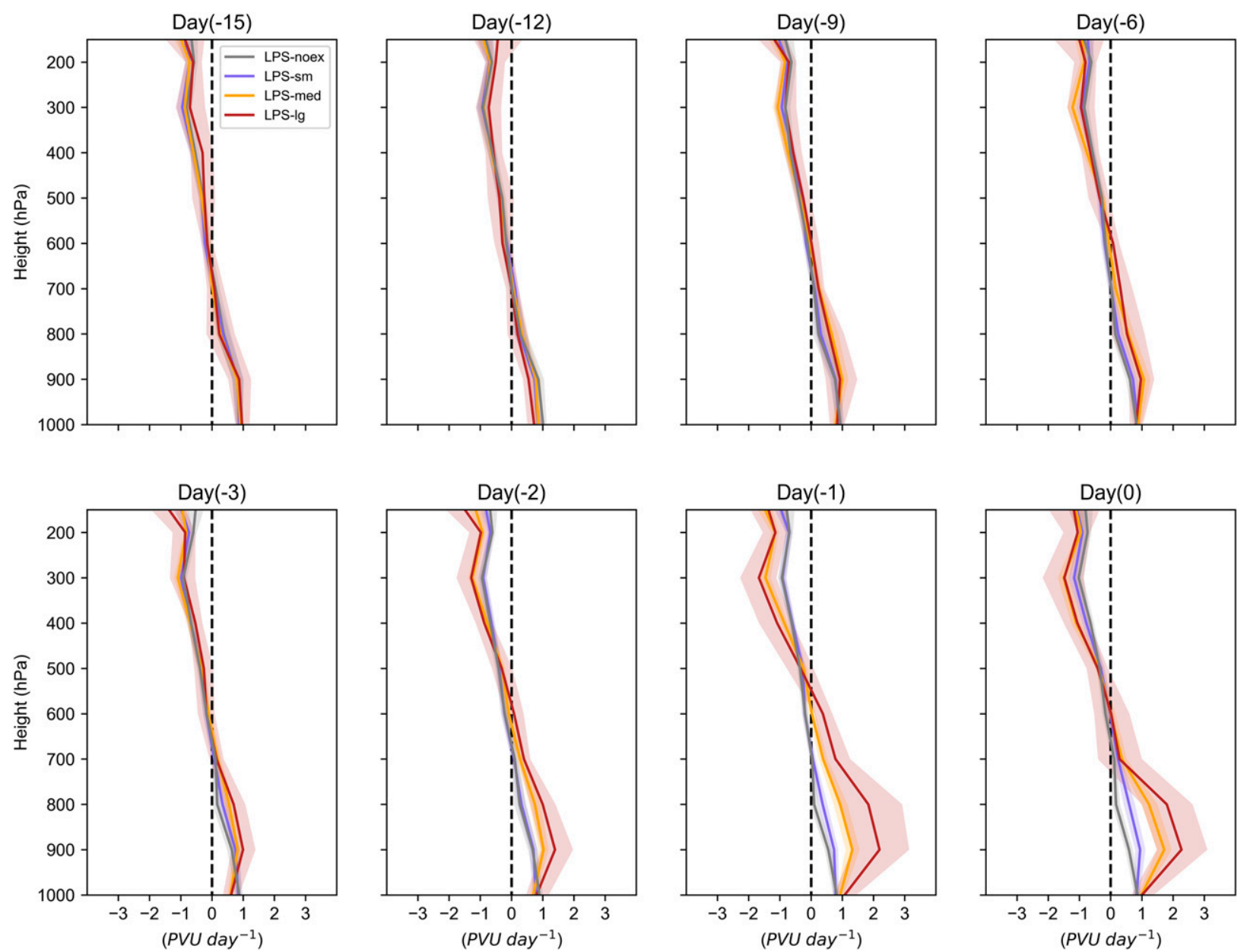

FIG. 10. As in Fig. 9, but for the diabatic PV tendency [obtained by Eq. (2)].

of LPSs and MISO that leads to EREs of various sizes. The spatial distributions of EREs of various sizes follow the spatial variations in the monsoon trough during the different phases of the MISO. The trough is situated over central India during the positive phase of the MISO and shifts near the foothills during the negative MISO phase. Accordingly, large and medium EREs occur mainly over the southern boundary $\left(15^{\circ}-20^{\circ} \mathrm{N}\right)$ of central India, while small EREs tend to occur near the northern boundary (north of $22^{\circ} \mathrm{N}$ ).

Processes (sections 3c-3e; Figs. 7-10) through which the phases of the MISO modulate the response of LPSs in producing EREs of different sizes are as follows.

1) During the positive phase of MISO, an active monsoon trough and a strong LLJ exist over central India. A large-scale dynamic forcing is set up due to enhanced temperature and vorticity gradients. When an LPS passes through central India it intensifies by vortex stretching as a strong convergence zone forms to the west of the LPS resulting from the interaction of the LLJ with the LPS. An active monsoon trough generates potential vorticity at low to midtroposphere through the diabatic effects and strengthens the low-level cyclonic circulation.
These conditions help in the organization of convection and LPSs produce medium and large EREs.

2) During the negative phase of MISO, large-scale dynamic subsidence exists at midlevels over central India associated with cold and negative vorticity anomalies. These LPSs do not intensify much during their lifetime. In the absence of an active monsoon trough, the low-level circulation is not strengthened via diabatic generation of potential vorticity. When an LPS passes through central India in such conditions, it triggers small EREs.

Here, we show how the interaction of the MISO phases with LPSs can modulate the spatial characteristics of EREs. Predicting the in-phase and out-of-phase conditions of multiple monsoon components can improve the forecast of different spatiotemporal characteristics of EREs. Such a forecast that includes the spatiotemporal characteristics could prove useful in preparing a proper action plan.

We use LPS-noex to bring out the contrast; however, further investigation is needed to understand them thoroughly and examine for the different spatiotemporal characteristics of wet events. A comprehensive study of the multiscale interactions of different monsoon components for all wet and dry events is 
essential. Similarly, temporal characteristics of wet and dry events in addition to spatial characteristics could be explored with a high-resolution dataset.

LPSs and MISO are modulated by other tropical variability like the Madden Julian oscillation (MJO) (Haertel and Boos 2017; Pai et al. 2011), and the slow-varying (interannual) modes like ENSO and the IOD (Ajayamohan et al. 2008; Yoo et al. 2010). Further efforts are needed to understand the influences of these large-scale drivers on the characteristics of EREs and thereby improve their prediction.

Acknowledgments. A. C. and G. S. B. acknowledge funding from the DST, MoES, and MoEF, Government of India. D. M. W. F. is supported by NSF Grant AGS-1665247. We thank S. Paleri and C. Jalihal for their inputs.

\section{REFERENCES}

Ajayamohan, R., S. A. Rao, and T. Yamagata, 2008: Influence of Indian Ocean dipole on poleward propagation of boreal summer intraseasonal oscillations. J. Climate, 21, 5437-5454, https://doi.org/10.1175/2008JCLI1758.1.

— W. W. Merryfield, and V. V. Kharin, 2010: Increasing trend of synoptic activity and its relationship with extreme rain events over central India. J. Climate, 23, 1004-1013, https://doi.org/ 10.1175/2009JCLI2918.1.

— B. Bhouider, A. J. Majda, and Q. Deng, 2016: Role of stratiform heating on the organization of convection over the monsoon trough. Climate Dyn., 47, 3641-3660, https://doi.org/ 10.1007/s00382-016-3033-7.

Allan, R. P., and B. J. Soden, 2008: Atmospheric warming and the amplification of precipitation extremes. Science, 321, 14811484, https://doi.org/10.1126/science.1160787.

Annamalai, H., and J. Slingo, 2001: Active/break cycles: Diagnosis of the intraseasonal variability of the Asian summer monsoon. Climate Dyn., 18, 85-102, https://doi.org/10.1007/s003820100161.

Boos, W., J. Hurley, and V. Murthy, 2015: Adiabatic westward drift of Indian monsoon depressions. Quart. J. Roy. Meteor. Soc., 141, 1035-1048, https://doi.org/10.1002/qj.2454.

Bretherton, C. S., M. E. Peters, and L. E. Back, 2004: Relationships between water vapor path and precipitation over the tropical oceans. J. Climate, 17, 1517-1528, https://doi.org/10.1175/15200442(2004)017<1517:RBWVPA > 2.0.CO;2.

Daggupaty, S. M., and D. R. Sikka, 1977: On the vorticity budget and vertical velocity distribution associated with the life cycle of a monsoon depression. J. Atmos. Sci., 34, 773-792, https://doi.org/ 10.1175/1520-0469(1977)034<0773:OTVBAV>2.0.CO;2.

Dee, D. P., and Coauthors, 2011: The ERA-Interim reanalysis: Configuration and performance of the data assimilation system. Quart. J. Roy. Meteor. Soc., 137, 553-597, https://doi.org/ 10.1002/qj.828.

Ding, Y., 2007: The variability of the Asian summer monsoon. J. Meteor. Soc. Japan, 85, 21-54, https://doi.org/10.2151/ jmsj.85B.21.

Emori, S., and S. Brown, 2005: Dynamic and thermodynamic changes in mean and extreme precipitation under changed climate. Geophys. Res. Lett., 32, L17706, https://doi.org/ 10.1029/2005GL023272.

Falcão, A. X., J. Stolfi, and R. de Alencar Lotufo, 2004: The image foresting transform: Theory, algorithms, and applications. IEEE Trans. Pattern Anal. Mach. Intell., 26, 19-29, https:// doi.org/10.1109/TPAMI.2004.1261076.
Francis, P., and S. Gadgil, 2006: Intense rainfall events over the west coast of India. Meteor. Atmos. Phys., 94, 27-42, https:// doi.org/10.1007/s00703-005-0167-2.

Godbole, R. V., 1977: The composite structure of the monsoon depression. Tellus, 29, 25-40, https://doi.org/10.3402/tellusa.v29i1.11327.

_ 1978: On cumulus-scale transport of horizontal momentum in monsoon depression over India. Monsoon Dynamics, Springer, 1373-1381.

Goswami, B., and R. A. Mohan, 2001: Intraseasonal oscillations and interannual variability of the Indian summer monsoon. J. Climate, 14, 1180-1198, https://doi.org/10.1175/ 1520-0442(2001)014<1180:IOAIVO>2.0.CO;2.

_, R. Ajayamohan, P. K. Xavier, and D. Sengupta, 2003: Clustering of synoptic activity by Indian summer monsoon intraseasonal oscillations. Geophys. Res. Lett., 30, https:// doi.org/10.1029/2002GL016734.

Haertel, P., and W. R. Boos, 2017: Global association of the Madden-Julian Oscillation with monsoon lows and depressions. Geophys. Res. Lett., 44, 8065-8074, https://doi.org/ 10.1002/2017GL073625.

Hazra, A., and V. Krishnamurthy, 2015: Space-time structure of diabatic heating in monsoon intraseasonal oscillation. J. Climate, 28, 2234-2255, https://doi.org/10.1175/JCLI-D-14-00280.1.

Holloway, C. E., and J. D. Neelin, 2009: Moisture vertical structure, column water vapor, and tropical deep convection. J. Atmos. Sci., 66, 1665-1683, https://doi.org/10.1175/ 2008JAS2806.1.

Holton, J., and G. Hakim, 2012: An Introduction to Dynamic Meteorology. Academic Press, 532 pp.

Hoskins, B., I. Draghici, and H. Davies, 1978: A new look at the w-equation. Quart. J. Roy. Meteor. Soc., 104, 31-38, https:// doi.org/10.1002/qj.49710443903.

Hurley, J. V., and W. R. Boos, 2015: A global climatology of monsoon low-pressure systems. Quart. J. Roy. Meteor. Soc., 141, 1049-1064, https://doi.org/10.1002/qj.2447.

Karmakar, N., A. Chakraborty, and R. S. Nanjundiah, 2015: Decreasing intensity of monsoon low-frequency intraseasonal variability over India. Environ. Res. Lett., 10, 054018, https:// doi.org/10.1088/1748-9326/10/5/054018.

Kiladis, G. N., C. D. Thorncroft, and N. M. Hall, 2006: Threedimensional structure and dynamics of African easterly waves. Part I: Observations. J. Atmos. Sci., 63, 2212-2230, https:// doi.org/10.1175/JAS3741.1.

Krishnamurthy, V., 2012: Extreme events and trends in the Indian summer monsoon. Extreme Events and Natural Hazards: The Complexity Perspective, Geophys. Monogr., Vol. 196, Amer. Geophys. Union, 153-168.

Krishnamurti, T. N., and H. Bhalme, 1976: Oscillations of a monsoon system. Part I. observational aspects. J. Atmos. Sci., 33, 1937-1954, https://doi.org/10.1175/1520-0469(1976)033<1937: OOAMSP $>2.0 . \mathrm{CO} ; 2$.

Ling, J., and C. Zhang, 2013: Diabatic heating profiles in recent global reanalyses. J. Climate, 26, 3307-3325, https://doi.org/ 10.1175/JCLI-D-12-00384.1.

Martin, J. E., 2006: Mid-Latitude Atmospheric Dynamics: A First Course. John Wiley \& Sons, $336 \mathrm{pp}$.

Moron, V., R. Barbero, H. J. Fowler, and V. Mishra, 2021: Storm types in India: Linking rainfall duration, spatial extent and intensity. Philos. Trans. Roy. Soc. London, A379, 20200137, https://doi.org/10.1098/rsta.2020.0137.

Nikumbh, A. C., A. Chakraborty, and G. Bhat, 2019: Recent spatial aggregation tendency of rainfall extremes over India. Sci. Rep., 9, 10321, https://doi.org/10.1038/s41598-019-46719-2. 
and D. M. Frierson, 2020: Large-scale extreme rainfall producing synoptic systems of the Indian summer monsoon. Geophys. Res. Lett., 47, e2020GL088403, https:// doi.org/10.1029/2020GL088403.

Pai, D., J. Bhate, O. Sreejith, and H. Hatwar, 2011: Impact of MJO on the intraseasonal variation of summer monsoon rainfall over India. Climate Dyn., 36, 41-55, https://doi.org/10.1007/s00382009-0634-4.

_ - L. Sridhar, and M. R. Kumar, 2016: Active and break events of Indian summer monsoon during 1901-2014. Climate Dyn., 46, 3921-3939, https://doi.org/10.1007/s00382-015-2813-9.

Patwardhan, S., K. P. Sooraj, H. Varikoden, S. Vishnu, K. Koteswararao, M. V. S. Ramarao, and D. R. Pattanaik, 2020: Synoptic scale systems. Assessment of Climate Change over the Indian Region, R. Krishnan et al., Eds., Springer, 143-154.

Phadtare, J., and G. Bhat, 2019: Characteristics of deep cloud systems under weak and strong synoptic forcing during the Indian summer monsoon season. Mon. Wea. Rev., 147, 37413758, https://doi.org/10.1175/MWR-D-18-0346.1.

Rajamani, S., and D. Sikdar, 1989: Some dynamical characteristics and thermal structure of monsoon depressions over the Bay of Bengal. Tellus, 41A, 255-269, https://doi.org/10.3402/tellusa.v41i3.11838.

Rajeevan, M., J. Bhate, J. Kale, and B. Lal, 2006: High resolution daily gridded rainfall data for the Indian region: Analysis of break and active. Curr. Sci., 91, 296-306, http://www.jstor.org/stable/24094135.

_- S. Gadgil, and J. Bhate, 2010: Active and break spells of the Indian summer monsoon. J. Earth Syst. Sci., 119, 229-247, https://doi.org/10.1007/s12040-010-0019-4.

Ramamurthy, K., 1969: Monsoon of India: Some aspects of the 'break' in the Indian southwest monsoon during July and August. Vol. 1, Forecasting Manual. Indian Meteorological Department, 1-57.

Rao, Y., 1976: Southwest monsoon. Synoptic Meteorology, India Meteorological Department, 90-91.

Roxy, M. K., S. Ghosh, A. Pathak, R. Athulya, M. Mujumdar, R. Murtugudde, P. Terray, and M. Rajeevan, 2017: A threefold rise in widespread extreme rain events over Central India. Nat. Commun., 8, 708, https://doi.org/10.1038/s41467-017-00744-9.

Sikka, D., 1977: Some aspects of the life history, structure and movement of monsoon depressions. Pure Appl. Geophys., 115, 1501-1529, https://doi.org/10.1007/BF00874421.
, and S. Gadgil, 1980: On the maximum cloud zone and the ITCZ over Indian, longitudes during the southwest monsoon. Mon. Wea. Rev., 108, 1840-1853, https://doi.org/10.1175/15200493(1980)108<1840:OTMCZA>2.0.CO;2.

Sillmann, J., and Coauthors, 2017: Understanding, modeling and predicting weather and climate extremes: Challenges and opportunities. Wea. Climate Extremes, 18, 65-74, https:// doi.org/10.1016/j.wace.2017.10.003.

Singh, D., M. Tsiang, B. Rajaratnam, and N. S. Diffenbaugh, 2014: Observed changes in extreme wet and dry spells during the South Asian summer monsoon season. Nat. Climate Change, $\mathbf{4}$, 456-461, https://doi.org/10.1038/nclimate2208.

_ S. Ghosh, M. K. Roxy, and S. McDermid, 2019: Indian summer monsoon: Extreme events, historical changes, and role of anthropogenic forcings. Wiley Interdiscip. Rev.: Climate Change, 10, e571, https://doi.org/10.1002/wcc.571.

Trenberth, K. E., A. Dai, R. M. Rasmussen, and D. B. Parsons, 2003: The changing character of precipitation. Bull. Amer. Meteor. Soc., 84, 1205-1218, https://doi.org/10.1175/BAMS84-9-1205.

Turner, A. G. and J. M. Slingo, 2009: Subseasonal extremes of precipitation and active-break cycles of the Indian summer monsoon in a climate-change scenario. Quart. J. Roy. Meteor. Soc., 135, 549-567, https://doi.org/10.1002/qj.401.

Wang, B., 2006: The Asian Monsoon. Springer, 787 pp.

Webster, P. J., V. O. Magana, T. Palmer, J. Shukla, R. Tomas, M. Yanai, and T. Yasunari, 1998: Monsoons: Processes, predictability, and the prospects for prediction. J. Geophys. Res., 103, 14 451-14 510, https://doi.org/10.1029/97JC02719.

Yanai, M., S. Esbensen, and J.-H. Chu, 1973: Determination of bulk properties of tropical cloud clusters from large-scale heat and moisture budgets. J. Atmos. Sci., 30, 611-627, https://doi.org/ 10.1175/1520-0469(1973)030<0611:DOBPOT > 2.0.CO;2.

Yasunari, T., 1979: Cloudiness fluctuations associated with the Northern Hemisphere summer monsoon. J. Meteor. Soc. Japan, 57, 227-242, https://doi.org/10.2151/jmsj1965.57.3_227.

Yoo, J. H., A. W. Robertson, and I.-S. Kang, 2010: Analysis of intraseasonal and interannual variability of the Asian summer monsoon using a hidden Markov model. J. Climate, 23, 5498-5516, https://doi.org/10.1175/2010JCLI3473.1. 